\title{
Caracterização termoanalítica do sulfato de cobre pentahidratado
}

\section{Thermoanalytical characterization of copper sulphate pentahydrate}

\author{
1 Lucas Rafael Carneiro da Silva Ircs.carneiro@gmail.com \\ 1 Railson Machado Pinto
}

1 Bacharel em Engenharia de Materiais, Mestrando - Universidade Federal do Piauí, Campus Ministro Petrônio Portella, Centro de Tecnologia.

\section{Resumo}

A análise térmica é o conjunto de técnicas, no qual uma propriedade física é medida durante um período de tempo em que a amostra é sujeitada a um programa controlado de temperatura. 0 presente trabalho, a partir de técnicas termoanalíticas, como a TG, DTG, DSC e DTA, avaliou a estabilidade térmica do Sulfato de Cobre Pentahidratado $\left(\mathrm{CuSO}_{4} \cdot 5 \mathrm{H}_{2} \mathrm{O}\right)$. $\mathrm{O}$ estudo de termodecomposição do $\mathrm{CuSO}_{4} \cdot 5 \mathrm{H}_{2} \mathrm{O}$ foi conduzido sob atmosfera dinâmica de Argônio (Ar) com o fluxo de $50 \mathrm{ml} / \mathrm{min}$, da temperatura ambiente até $1000^{\circ} \mathrm{C}$ e com a razão de aquecimento de $10{ }^{\circ} \mathrm{C} / \mathrm{min}$. Com base nos resultados obtidos, pode-se concluir, para a análise por TG e DTG, que a termodecomposição do $\mathrm{CuSO}_{4} \cdot 5 \mathrm{H}_{2} \mathrm{O}$ ocorreu em seis eventos térmicos, cada um relacionado à saída de uma molécula e indicado por uma perda de massa. Todos os picos da análise por DSC e DTA tiveram o caráter endotérmico. As técnicas se mostraram satisfatórias na caracterização térmica do material.

\section{Palavras-chave}

Análise térmica. Caracterização. Termodecomposição. Termogravimetria.

\begin{abstract}
Thermal analysis is the set of techniques in which a physical property is measured over a period of time when the sample is subjected to a controlled temperature program. The present work based on thermoanalytical techniques, such as TG, DTG, DSC and DTA, evaluated the thermal stability of Copper Sulphate Pentahydrate (CuSO $\left.{ }_{4} \cdot 5 \mathrm{H}_{2} \mathrm{O}\right)$. The thermodecomposition study of $\mathrm{CuSO}_{4} .5 \mathrm{H}_{2} \mathrm{O}$ was conducted under dynamic Argon (Ar) atmosphere with a flow rate of $50 \mathrm{ml} / \mathrm{min}$, from room temperature to $1000{ }^{\circ} \mathrm{C}$ and with a heating ratio of $10{ }^{\circ} \mathrm{C} / \mathrm{min}$. Based on the results obtained, it can be concluded for TG and DTG analysis that the thermodecomposition of $\mathrm{CuSO}_{4} \cdot 5 \mathrm{H}_{2} \mathrm{O}$ occurred in six thermal events, each related to the output of a molecule and indicated by a mass loss. All peaks of the DSC and DTA analysis were endothermic. The techniques were satisfactory in the thermal characterization of the material.
\end{abstract}

\section{Keywords}

Thermal analysis. Characterization.

Thermodecomposition. Thermogravimetry.

\section{Como você deve citar?}

SILVA, Lucas Rafael Carneiro; PINTO, Railson Machado. Caracterização termoanalítica do sulfato de cobre pentahidratado.Cadernos UniFOA, Volta Redonda, n. 43, p. 25-31, agosto 2020. 


\section{INTRODUÇÃO}

De acordo com Ozawa (2000), a análise térmica já teve mais de 11 décadas de história. 0 autor afirma que, no decorrer dessa extensa história, diversas técnicas foram desenvolvidas e a análise térmica foi sendo aplicada em inúmeras áreas de estudo. Destaca-se que, atualmente, a utilização da análise térmica se difunde entre os minerais, as cerâmicas, os metais, os polímeros, os produtos farmacêuticos, os materiais eletrônicos, os organismos biológicos etc.

Uma definição bastante difundida para a análise térmica aborda que se trata de um conjunto de técnicas, no qual uma propriedade física é medida durante um período de tempo em que a amostra é sujeitada a um programa controlado de temperatura. Podem ser obtidas através dessas técnicas informações correspondentes à variação de massa, estabilidade térmica, calor específico, grau de hidratação, cinética de reação etc. (CAVALHEIRO et al., 1995; BANNACH et al., 2011).

A caracterização de uma técnica térmica como sendo termoanalítica sujeita-se à condição de que é fundamental abranger a medição de uma propriedade física, de maneira direta ou indireta na dependência da temperatura sob um programa controlado (SILVA et al., 2007). As técnicas mais empregadas são: Termogravimetria (TG), Termogravimetria Derivada (DTG), Calorimetria Exploratória Diferencial (DSC) e Análise Térmica Diferencial (DTA) (GIOLITO; IONASHIRO, 1988). 0 uso dessas técnicas em muitos campos de estudo fez com que elas fossem caracterizadas como ferramentas interdisciplinares, sendo úteis na ciência e tecnologia (IONASHIRO, 2004). Perante o exposto, o objetivo deste trabalho foi investigar a estabilidade térmica do Sulfato de Cobre Pentahidratado $\left(\mathrm{CuSO}_{4} \cdot 5 \mathrm{H}_{2} \mathrm{O}\right)$ empregando-se as técnicas mencionadas.

\section{MATERIAIS E MÉTODOS}

O material utilizado no trabalho foi o Sulfato de Cobre Pentahidratado $\left(\mathrm{CuSO}_{4} \cdot 5 \mathrm{H}_{2} \mathrm{O}\right)$. A caracterização termoanalítica foi realizada em um equipamento SDT Q600 V20.9 Build 20 (TA Instruments), no Laboratório Interdisciplinar de Materiais Avançados (LIMAV) da Universidade Federal do Piauí (UFPI), sob atmosfera dinâmica de Argônio (Ar) com o fluxo de $50 \mathrm{ml} / \mathrm{min}$, da temperatura ambiente até 1000 ${ }^{\circ} \mathrm{C}$ e com a razão de aquecimento de $10{ }^{\circ} \mathrm{C} / \mathrm{min}$. A massa da amostra usada foi de $6,49 \mathrm{mg}( \pm 0,1 \mathrm{mg})$, medida em um suporte aberto de a-Alumina ligado à termobalança. A análise foi realizada sob pressão atmosférica e o módulo TG foi previamente calibrado, segundo as instruções do fabricante. Na Figura 1, é ilustrada a estrutura molecular do $\mathrm{CuSO}_{4} \cdot 5 \mathrm{H}_{2} \mathrm{O}$.

Figura 1 - Estrutura molecular do $\mathrm{CuSO}_{4} \cdot 5 \mathrm{H}_{2} \mathrm{O}$.

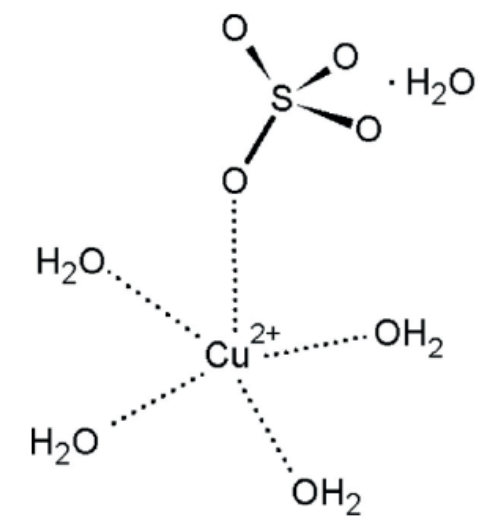

Fonte: adaptado de Lavrenyuk et al. (2016). 


\section{$3 \quad$ RESULTADOS E DISCUSSÃO}

A curva TG [Massa (\%)] e DTG [Derivada da Massa $\left(\% /{ }^{\circ} \mathrm{C}\right)$ ] obtida para o $\mathrm{CuSO}_{4} \cdot 5 \mathrm{H}_{2} \mathrm{O}$ é apresentada na Figura 2. Essas curvas mostraram a decomposição da amostra em seis etapas, com diferenças nas perdas de massa. As etapas ao longo da análise foram caracterizadas por seis eventos térmicos, descritos por patamares na curva TG e por picos na curva DTG.

Figura 2 - Curva TG (\%) e DTG $\left(\% /{ }^{\circ} \mathrm{C}\right)$ do $\mathrm{CuSO}_{4} \cdot 5 \mathrm{H}_{2} \mathrm{O}$ sob atmosfera dinâmica de Ar.

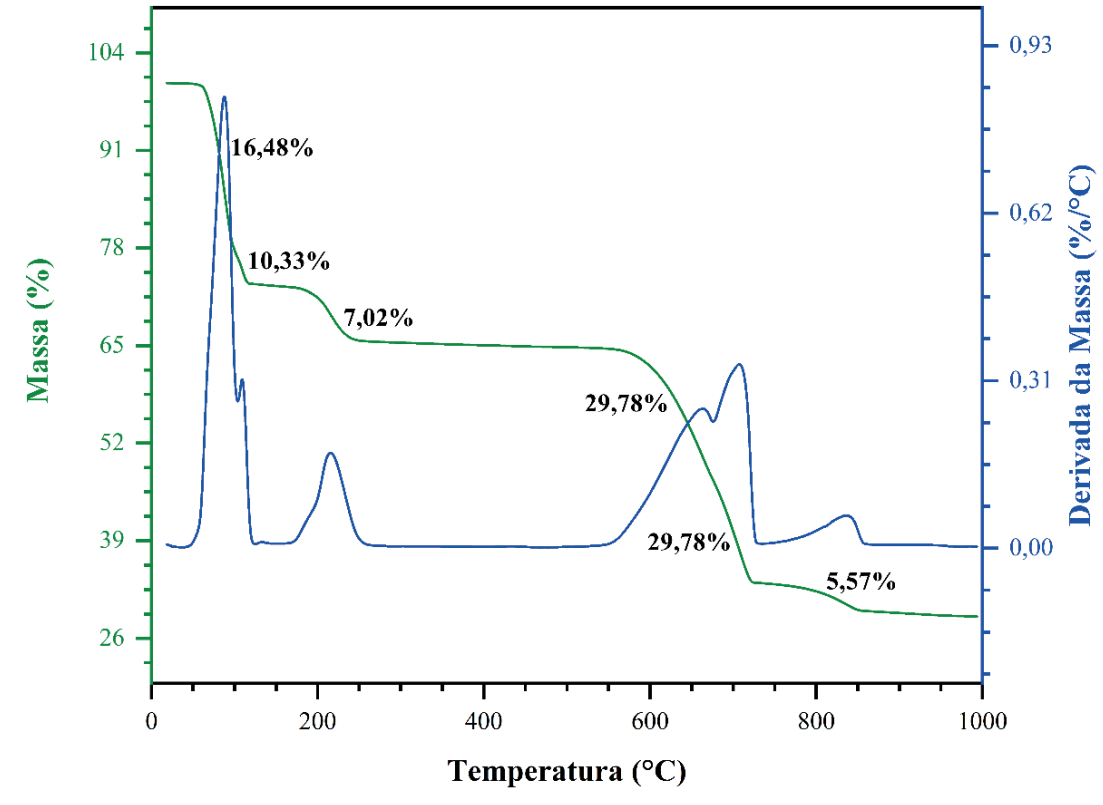

Fonte: autores, 2020

Verificou-se, na curva TG da amostra, em um $1^{\circ}$ evento, que ocorreu a eliminação de duas moléculas de Água $\left(2 \mathrm{H}_{2} \mathrm{O}\right)$ com a perda de massa em torno de $16,48 \%$ entre a Temperatura Inicial $\left(\mathrm{T}_{\mathrm{i}}\right)$ de $50,02^{\circ} \mathrm{C}$ e Temperatura Final $\left(T_{f}\right)$ de $90,20^{\circ} \mathrm{C}$. Determinou-se os valores de Temperatura Onset ( $\left.T_{\text {onset }}\right)$ igual a 68,98 ${ }^{\circ} \mathrm{C}$, que correspondeu ao início extrapolado do evento térmico, e Temperatura Endset $\left(\mathrm{T}_{\text {endset }}\right.$ ), resultando em $89,91^{\circ} \mathrm{C}$ sendo, dessa maneira, a temperatura final do evento extrapolado. Apurou-se que o primeiro pico na curva DTG equiparou-se a essa perda de $2 \mathrm{H}_{2} \mathrm{O}$, tendo em vista o valor de Temperatura Máxima $\left(T_{\text {máx }}\right)$ ao redor de $87,60{ }^{\circ} \mathrm{C}$ e valores de $T_{\text {onset }}$ e $T_{\text {endset' }}$ respectivamente, 57,21 e $104,86^{\circ} \mathrm{C}$.

No $2^{\circ}$ evento, notou-se a decomposição de Sulfato de Cobre Trihidratado ( $\left.\mathrm{CuSO}_{4} \cdot 3 \mathrm{H}_{2} \mathrm{O}\right)$, gerado na etapa anterior com a perda de massa, cerca de 10,33\% em um intervalo de temperatura de $90,27-144,16{ }^{\circ} \mathrm{C}$, e também houve a eliminação de $2 \mathrm{H}_{2} \mathrm{O}$ (Roca, 2007). Os valores de $\mathrm{T}_{\text {onset }}$ e $\mathrm{T}_{\text {endset }}$ para a curva TG resultaram em 103,21 e $131,22^{\circ} \mathrm{C}$, de modo respectivo, e para a curva DTG os resultados conseguidos, foram de $79,96{ }^{\circ} \mathrm{C}$ para a $\mathrm{T}_{\text {onset }}$ e $118,82^{\circ} \mathrm{C}$ para a $\mathrm{T}_{\text {endsett }}$ e a $\mathrm{T}_{\text {máx }}$; para o segundo pico, foi por volta de $109,13^{\circ} \mathrm{C}$.

Em um $3^{\circ}$ evento, ocorreu a perda de massa por volta de $7,02 \%$ entre $179,33-259,93^{\circ} \mathrm{C}$, que se relacionou à liberação de uma molécula de $\mathrm{H}_{2} \mathrm{O}$ proveniente da decomposição de Sulfato de Cobre Monohidratado ( $\mathrm{CuSO}_{4} \cdot \mathrm{H}_{2} \mathrm{O}$ ) (El-Houte et al., 1989). Os valores de $\mathrm{T}_{\text {onset }}$ e $\mathrm{T}_{\text {endset }}$ foram de 195,60 e 234,08 ${ }^{\circ} \mathrm{C}$ para a TG e de 188,18 e $248,24^{\circ} \mathrm{C}$ para a DTG. $O$ resultado atingido para a $\mathrm{T}_{\text {máx }}$ do terceiro pico na curva DTG foi de $215,48^{\circ} \mathrm{C}$. 
$04^{\circ}$ e o $5^{\circ}$ eventos apresentaram a liberação de uma molécula de Óxido Sulfúrico $\left(\mathrm{SO}_{3}\right)$ com a perda de massa de $29,78 \%$ para ambos na faixa de temperatura de $530-716,05^{\circ} \mathrm{C}$. Os valores para a curva TG de $\mathrm{T}_{\text {onset }}$ e $\mathrm{T}_{\text {endset }}$ foram, respectivamente, 610,61 e $721,60^{\circ} \mathrm{C}$; já para a curva $\mathrm{DTG}$, as temperaturas resultaram em 567,95 e $726,03^{\circ} \mathrm{C}$ ( $4^{\circ}$ evento) e 629 e $726,08^{\circ} \mathrm{C}$ ( $5^{\circ}$ evento). 0 resultado alcançado para a $\mathrm{T}_{\text {máx }}$ do quarto e quinto pico na curva DTG foi, na devida ordem, 663,89 e $707,55^{\circ} \mathrm{C}$.

No $6^{\circ}$ evento os dados obtidos foram: perda de massa de $5,57 \%$; faixa de temperatura de 716,22 a $911,60{ }^{\circ} \mathrm{C}$; $\mathrm{T}_{\text {onset }}$ igual a $786,12^{\circ} \mathrm{C}$ e $\mathrm{T}_{\text {endset' }}$ correspondente a $846,91^{\circ} \mathrm{C}$ (curva TG). No caso da curva DTG, obteve-se a $\mathrm{T}_{\text {onset }}$ de $771,33^{\circ} \mathrm{C}$ e $\mathrm{T}_{\text {endset }}$ no valor de $855,94^{\circ} \mathrm{C}$, e o resultado alcançado para a $\mathrm{T}_{\text {máx }}$ do sexto pico na curva DTG representativo da eliminação de $1 / 2 \mathrm{O}_{2}$ foi de $840^{\circ} \mathrm{C}$. A $993,24{ }^{\circ} \mathrm{C}$, a amostra apresentou um resíduo de $30 \% \mathrm{com}$ a massa residual de $1,876 \mathrm{mg}$, que correspondeu a um produto final de Óxido de Cobre $\left(\mathrm{Cu}_{2} \mathrm{O}\right)$.

Aparentemente na curva TG pode parecer que houve apenas quatro eventos térmicos, mas, como supracitado, foram seis eventos. No início da decomposição do $\mathrm{CuSO}_{4} \cdot 5 \mathrm{H}_{2} \mathrm{O}$, obteve-se dois eventos que ficaram bem juntos $\left(1^{\circ}\right.$ e $\left.2^{\circ}\right)$, do mesmo modo que a maior decomposição da curva também apresentou dois eventos muito próximos $\left(4^{\circ}\right.$ e $\left.5^{\circ}\right)$. Aqui, ressalta-se a importância do que foi mencionado logo acima: mesmo que os resultados da curva TG tenham expressado uma perda de massa bem definida para alguns eventos $\left(3^{\circ}\right.$ e $\left.6^{\circ}\right)$, para outros eventos, visualizar a perda tornou-se bem mais difícil. Todavia, a curva DTG exibiu-se mais bem definida e, assim, os seis eventos foram visualizados com uma maior precisão.

Roca (2007) analisou o CuSO ${ }_{4} \cdot 5 \mathrm{H}_{2} \mathrm{O}$ sob atmosfera de Nitrogênio $\left(\mathrm{N}_{2}\right)$ com o fluxo de $50 \mathrm{ml} / \mathrm{min}$ e razão de aquecimento de $10^{\circ} \mathrm{C} / \mathrm{min}$ e obteve a curva TG similar à curva alcançada neste trabalho, apesar da atmosfera utilizada ter sido diferente. De acordo com White (2012), os mecanismos de desidratação térmica do $\mathrm{CuSO}_{4} \cdot 5 \mathrm{H}_{2} \mathrm{O}$ têm sido o objeto de numerosos estudos ao longo dos últimos 80 anos. $\mathrm{O}$ autor afirma que a taxa de perda de água do $\mathrm{CuSO}_{4} \cdot 5 \mathrm{H}_{2} \mathrm{O}$ quando aquecido, é fortemente dependente dos parâmetros experimentais. Conforme Topley e Smith (1931), a desidratação do $\mathrm{CuSO}_{4} \cdot 5 \mathrm{H}_{2} \mathrm{O}$ também é afetada pela concentração do vapor de água no ambiente. Ademais, outros estudos indicam ainda que a taxa de desidratação depende do tamanho das partículas e, também, da razão de aquecimento (TAYLOR; KLUG, 1936; NAGASE ET AL., 1980). Na Tabela 1, são apresentados os Intervalos de Temperatura em que ocorreram os eventos térmicos para a sequência da decomposição do $\mathrm{CuSO}_{4} \cdot 5 \mathrm{H}_{2} \mathrm{O}$, a Perda de Massa e o Resíduo.

Tabela 1 - Resultados obtidos por meio da curva TG do $\mathrm{CuSO}_{4} \cdot 5 \mathrm{H}_{2} \mathrm{O}$.

\begin{tabular}{ccc}
\hline Evento & Intervalo de Temperatura $\left({ }^{\circ} \mathbf{C}\right)$ & Perda de Massa (\%) \\
\hline $\mathbf{1}$ & $50,02-90,20$ & 16,48 \\
\hline $\mathbf{2}$ & $90,27-144,16$ & 10,33 \\
\hline $\mathbf{3}$ & $179,33-259,93$ & 7,02 \\
\hline $\mathbf{4}$ & $530-716,05$ & 29,78 \\
\hline $\mathbf{5}$ & $530-716,05$ & 29,78 \\
\hline $\mathbf{6}$ & $716,22-911,60$ & 5,57 \\
\hline Resíduo & 993,24 & 30 \\
\hline
\end{tabular}

Fonte: autores, 2020.

A curva DSC/DTA [Fluxo de Calor ( $\mathrm{mW}) /$ Diferença de Temperatura $(\mu \mathrm{V})$ ] é exibida na Figura 3. Observou-se, por meio das curvas, resultados com seis eventos endotérmicos mostrados como picos. 
Figura 3 - Curva DSC $(\mathrm{mW})$ e DTA $(\mu \mathrm{V})$ do $\mathrm{CuSO}_{4} \cdot 5 \mathrm{H}_{2} \mathrm{O}$ sob atmosfera dinâmica de Ar.

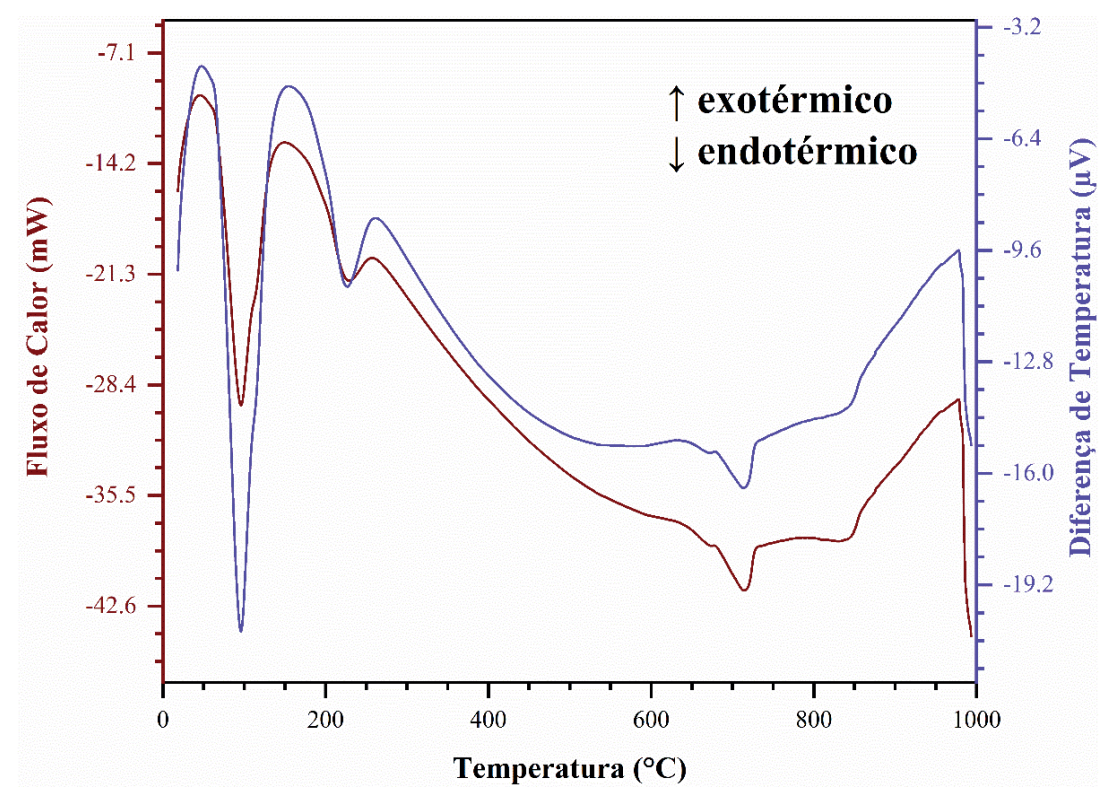

Fonte: autores, 2020

$01^{\circ}$ e $2^{\circ}$ eventos da curva DSC refere-se à desidratação do CuSO ${ }_{4} \cdot 5 \mathrm{H}_{2} \mathrm{O}$ com a eliminação de $2 \mathrm{H}_{2} \mathrm{O}$ na faixa de temperatura de $100,06-105,30^{\circ} \mathrm{C}$ e $106,01-119,92^{\circ} \mathrm{C}$, respectivamente. Os resultados são similares no trabalho de Lavrenyuk et al. (2016), em que, verificou que o primeiro estágio da degradação térmica envolve a faixa de temperatura de $20-190^{\circ} \mathrm{C}$ por meio da curva de DTA e que, nessa faixa, surge o efeito endotérmico com o valor extremo de temperatura a $120^{\circ} \mathrm{C}$, onde essa faixa da curva concerne ao processo de desidratação da água de cristalização e, em seguida, da água coordenada. $03^{\circ}$ evento exibiu o pico entre $200,04-300,01^{\circ} \mathrm{C}$ (DSC).

No $4^{\circ}, 5^{\circ}$ e $6^{\circ}$ eventos, os picos foram definidos, respectivamente, na faixa de temperatura de $643,50-675,96^{\circ} \mathrm{C} ; 720,49-731,01^{\circ} \mathrm{C}$ e $750,27-830,05^{\circ} \mathrm{C}$, para a curva DSC. Na Tabela 2, é mostrado a $\mathrm{T}_{\text {máx }}$ e Entalpia (curva DSC) e a $\mathrm{T}_{\text {onset' }} \mathrm{T}_{\text {endset }}$ e $\mathrm{T}_{\text {máx }}$ (curva DTA).

Tabela 2 - Resultados obtidos por meio da curva DSC e DTA do $\mathrm{CuSO}_{4} \cdot 5 \mathrm{H}_{2} \mathrm{O}$.

\begin{tabular}{|c|c|c|c|c|c|c|}
\hline \multirow{3}{*}{ Temperatura $\left({ }^{\circ} \mathrm{C}\right)$} & \multicolumn{6}{|c|}{ Evento } \\
\hline & $1^{\circ}$ & $2^{\circ}$ & $3^{\circ}$ & $4^{\circ}$ & $5^{\circ}$ & $6^{\circ}$ \\
\hline & \multicolumn{6}{|c|}{ DSC } \\
\hline $\mathbf{T}_{\text {máx }}$ & 99,75 & 115,41 & 222,61 & 659,89 & 716,50 & 842,03 \\
\hline \multirow[t]{2}{*}{ Entalpia (k.J/mol) } & \multicolumn{2}{|c|}{97,58} & 228,98 & & 714,48 & \\
\hline & \multicolumn{6}{|c|}{ DTA } \\
\hline $\mathbf{T}_{\text {onset }}$ & 61,31 & 107,97 & 205,06 & 637,19 & 636,52 & 798,26 \\
\hline $\mathbf{T}_{\text {endset }}$ & 135,30 & 122,63 & 261,49 & 678,73 & 780,95 & 754,69 \\
\hline $\mathbf{T}_{\text {máx }}$ & 97,31 & 111,97 & 225,06 & 675,25 & 731,61 & 844,47 \\
\hline
\end{tabular}

Fonte: autores, 2020.

A entalpia de desidratação é um fenômeno químico em que o calor é absorvido do sistema, no qual essa situação foi descrita pelo primeiro pico negativo da curva DSC/DTA correspondente ao $1^{\circ}$ evento endotérmico. Foi observado que o valor de entalpia que caracteriza a perda de $4 \mathrm{H}_{2} \mathrm{O}$ da amostra 
diz respeito ao resultado da integral da junção do $1^{\circ}$ e $2^{\circ}$ eventos, resultando em um valor de $97,58 \mathrm{k} . \mathrm{J} /$ mol. $03^{\circ}$ evento obteve o valor de $228,98 \mathrm{k} . \mathrm{J} / \mathrm{mol}$, e as entalpias do $4^{\circ}, 5^{\circ}$ e $6^{\circ}$ eventos foram somadas, sendo obtido o valor de $714,48 \mathrm{k} . \mathrm{J} / \mathrm{mol}$.

\section{CONCLUSÕES}

Com base nos resultados obtidos, pode-se concluir, para a análise por TG e DTG, que a termodecomposição do $\mathrm{CuSO}_{4} \cdot 5 \mathrm{H}_{2} \mathrm{O}$ ocorreu em seis eventos térmicos, cada um relacionado à saída de uma molécula e indicado por uma perda de massa. Todos os picos da análise por DSC e DTA apresentaram o caráter endotérmico. As técnicas se mostraram bastante satisfatórias na caracterização térmica do material.

\section{AGRADECIMENTOS}

À UFPI, ao Curso de Bacharelado em Engenharia de Materiais (Centro de Tecnologia - UFPI) e ao LIMAV - UFPI.

\section{REFERÊNCIAS}

BANNACH, G.; PERPÉTUO, G. L.; CAVALHEIRO, É. T. G.; CAVALHEIRO, C. C. S.; ROCHA, R. R. Efeitos da história térmica nas propriedades do polímero pet: um experimento para ensino de análise térmica. Química Nova, v. 34, n. 10, p. 1825-1829, 2011. Disponível em: https://doi.org/10.1590/S0100-40422011001000016. Acesso em: 15 out. 2019.

CAVALHEIRO, É. T. G.; IONASHIRO, M.; BREVIGLIERI, S. T.; MARINO, G.; CHIERICE, G. O. A Influência de Fatores Experimentais nos Resultados de Análises Termogravimétricas. Química Nova, v. 18, n. 3, p. 305-308, 1995. Acesso em: 15 out. 2019.

EL-HOUTE, S.; ALI, M. E. S.; SøRENSEN, O. T. Dehydration of $\mathrm{CuSO}_{4} \cdot 5 \mathrm{H}_{2} \mathrm{O}$ studied by conventional and advanced thermal analysis techniques. Thermochimica Acta, v. 138, n. 1, p. 107-114, 1989. Disponível em: https://doi.org/10.1016/0040-6031(89)87245-4. Acesso em: 18 out. 2019.

GIOLITO, I.; IONASHIRO, M. A nomenclatura em análise térmica. Parte II. Cerâmica, v. 34, n. 225, p. 1634, 1988. Acesso em: 16 out. 2019.

IONASHIRO, M. Princípios Básicos da Termogravimetria e Análise Térmica Diferencial/Calorimetria Exploratória Diferencial. São Paulo: Giz Editorial, 2004.

LAVRENYUK, H.; KOCHUBEI, V.; MYKHALICHKO, O.; MYKHALICHKO, B. A new flame retardant on the basis of diethylenetriamine copper(II) sulfate complex for combustibility suppressing of epoxy-amine composites. Fire Safety Journal, v. 80, p. 30-37, 2016. Disponível em: https://doi.org/10.1016/j.firesaf.2016.01.001. Acesso em: 20 out. 2019.

NAGASE, K.; YOKOBAYASHI, H.; KIKUCHI, M.; SONE, K. Effects of heating rate $\left(1-300^{\circ} \mathrm{h}^{-1}\right)$ on the nonisothermal thermogravimetry of $\mathrm{CuSO}_{4} \cdot 5 \mathrm{H}_{2} \mathrm{O}$. Thermochimica Acta, v. 35, n. 1, p. 99-104, 1980. Disponível em: https://doi.org/10.1016/0040-6031(80)85027-1. Acesso em: 19 out. 2019. 
OZAWA, T. Thermal analysis - review and prospect. Thermochimica Acta, v. 355, n. 1-2, p. 35-42, 2000. Disponível em: https://doi.org/10.1016/S0040-6031(00)00435-4. Acesso em: 15 out. 2019.

ROCA, Jesús Martínez. Influencia de variables experimentales seleccionadas en la descomposición térmica de $\mathrm{CaC}_{2} \mathrm{O}_{4} \cdot \mathrm{H}_{2} \mathrm{O}$ y $\mathrm{CuSO}_{4} \cdot 5 \mathrm{H}_{2} \mathbf{O} .2007$. Proyecto Fin de Carrera (Ingeniero Técnico Industrial) Universidad Politécnica de Cartagena, Cartagena, 2007.

SILVA, E. C.; PAOLA, M. V. R. V.; MATOS, J. R. Análise térmica aplicada à cosmetologia. Revista Brasileira de Ciências Farmacêuticas, v. 43, n. 3, p. 347-356, 2007. Disponível em: https://doi.org/10.1590/S151693322007000300004. Acesso em: 16 out. 2019.

TAYLOR, T. I.; KLUG, H. P. Thermal Transitions in Copper Sulphate Pentahydrate Molecular Rotation and the Dehydration of Hydrates. The Journal of Chemical Physics, v. 4, n. 9, p. 601-607, 1936. Disponível em: https://doi.org/10.1063/1.1749912. Acesso em: 19 out. 2019.

TOPLEY, B.; SMITH, M. L. Function of Water Vapour in the Dissociation of a Salt Hydrate. Nature, v. 128, p. 302, 1931. Disponível em: https://doi.org/10.1038/128302a0. Acesso em: 19 out. 2019.

WHITE, R. L. Variable temperature infrared study of copper sulfate pentahydrate dehydration. Thermochimica Acta, v. 528, p. 58-62, 2012. Disponível em: https://doi.org/10.1016/j.tca.2011.11.013. Acesso em: 19 out. 2019. 